\title{
Analysis of Plasma Treatment Effects on a Compliant Substrate for High Conductive, Stretchable Ag Nanowires
}

\author{
Jonghyun Jeong and Jaewook Jeong* \\ School of Information and Communication Engineering, Chungbuk National University, Cheongju 28644, Korea
}

Received December 19, 2017; revised January 12, 2018; accepted January 25, 2018

\begin{abstract}
In this paper, plasma treatment effects on a ploy(dimethyl siloxane) substrate were analyzed for the applications of stretchable silver nanowire (Ag NWs) electrodes. The oxygen plasma treated sample shows the best performance compared to nitrogen treated and untreated samples. The lowest sheet resistance and reasonable stretching capability was achieved up to $20 \%$ strain condition without open circuit fail for the oxygen plasma treated sample.
\end{abstract}

Keywords: plasma, PDMS, Ag NWs, stretchable electrode

\section{Introduction}

Plasma treatments on various substrates are widely used to obtain proper surface wetting properties for active and passive device applications such as stretchable electrodes and thin-film transistors. In particular, it is typically understood that the plasma treatments can modify the surface wetting properties from hydrophobic to hydrophilic states by lowering surface energy [1,2]. In some applications, the wettability of the surface is a critical issue to fabricate thin films on the substrate. In example, an active layer of solution-processed thin-film transistor is fabricated by spin coating on glass or Si substrates [3]. In this case, high wettability and low surface energy of the target substrate should be satisfied to obtain uniform and high quality semiconductor layers. Another important example is the formation of conductive electrodes on flexible or elastomeric substrates using water soluble materials such as nanowires (NWs). The method is widely used to form high conductive and transparent electrodes on a substrate employing various solution-based processes including inkjet printing and spray coating [4-8]. Compared to Si or glass substrates, the surface of flexible or elastomeric substrate i.e. PI or PET plastic and poly (dimethyl siloxane) (PDMS) substrates show very high hydrophobicity with high surface energy [5,9]. Therefore, it is impossible to form the uniform conductive electrode without any surface treatments. Therefore, up to now, various surface treatment methods have been investigated. Among the various methods, plasma treatment has been mainly used because it is simple, compatible with Si-based

*Corresponding author

E-mail: jjeong@cbnu.ac.kr process, and cost-effective compared to other method. In addition, it is typically understood that the plasma treatments are performed using oxygen plasma to generate hydroxyl group (OH-) on the surface of a rigid substrate. Also, it is known that hydroxyl group improves the adhesion property between two layers; i.e. rigid substrate and solution. Likewise, after plasma treatments on the surface of the PDMS substrate, the hydroxyl group is significantly increased, which is expected to improve the quality of spray-coated Ag NWs

In this paper, we introduce the plasma treatment methods on the PDMS substrate to fabricate the silver NW electrodes with high stretching capability. For the comparison of the effects of the plasma treatment, $\mathrm{O}_{2}$ and $\mathrm{N}_{2}$ plasma were applied to the surface of the PDMS substrate. We found that the $\mathrm{O}_{2}$ plasma treated sample shows the best performance due to high wettability. The performance of each sample was analyzed as a function of a strain.

\section{Experiments}

The elastomeric PDMS substrates were prepared using Sylgard-184 polymer (from Dow Conring Inc.) with a cross-linker (10:1 ratio of base to cross-linker). Then, the plasma treatments were applied to the prepared PDMS substrate. The plasma treatments were formed using a plasma system from Femto Science Inc. (Model No.: CUTE-1MPR). Two plasma species of $\mathrm{O}_{2}$ and $\mathrm{N}_{2}$ were applied to the prepared PDMS substrate. High purity process gases of $\mathrm{N}_{2}$ (99.99\% purity) and $\mathrm{O}_{2}(99.99 \%$ purity) were provided to the chamber for the generation of ion plasma. The process conditions of the plasma treatments were as follows: base pressure: $5 \times 10^{-3}$ torr, power: $80 \mathrm{~W}$, process pressure: 1.4 torr. The power and 
(a)

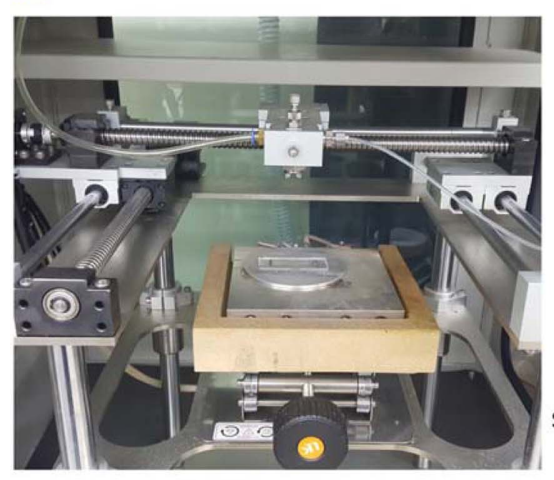

(b)

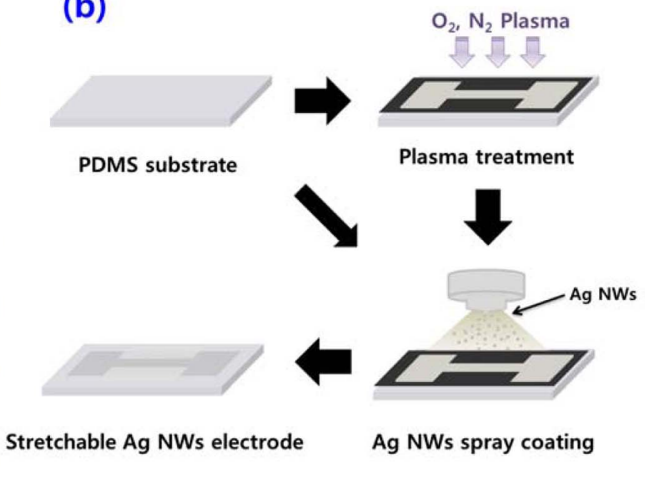

(c)

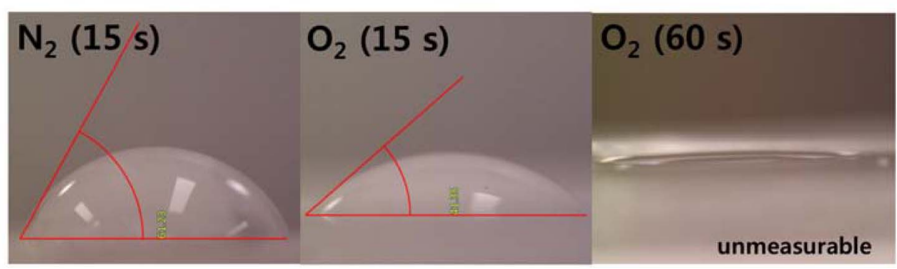

Figure 1. (a) A picture of spray coater set up. (b) Detailed process steps of Ag NW spray coating. (c) DI water droplet images on the PDMS substrates of $\mathrm{N}_{2}$-treated (15 s, left), $\mathrm{O}_{2}$-treated (15 s, center), and $\mathrm{O}_{2}$-treated (60 s, right) samples.

pressure were determined as typical range of the process condition for plasma treatments on the PDMS substrates. The flow rates of $\mathrm{O}_{2}$ and $\mathrm{N}_{2}$ gases were 76 and $95 \mathrm{sccm}$, respectively, which is dependent on the process pressure. The process time were optimized by measurements of deionized (DI) water contact angle. Then the high purity Ag NW solution (purchased from Plasmachem) was prepared, which contains Polyvinylpyrrolidone (PVP) stabilizer. The solution was diluted in DI water at a ratio of $10: 1$. The solution was spray coated on the prepared PDMS substrate through shadow mask at a substrate temperature of $120^{\circ} \mathrm{C}$ using a custom-made spray-coater shown in Fig. 1(a). All the process steps were summarized in Figure 1(b). The $\mathrm{O}_{2}$-treated $\left(\mathrm{O}_{2}\right.$ sample), $\mathrm{N}_{2}$-treated $\left(\mathrm{N}_{2}\right.$ sample), and reference (no treatment) samples were loaded to a custom-made stretching machine. In situ measurements of the resistance variations were performed under the stretching conditions using Agilent 34450A.

\section{Results and Discussion}

Firstly, we measured surface contact angle of deionized (DI) water on the reference, $\mathrm{N}_{2}$ and $\mathrm{O}_{2}$ plasma treated substrates, respectively. Figure 1(c) shows the DI water droplet images on the PDMS substrates depending on the process time and the plasma sources. For the $\mathrm{O}_{2}$ sample, the contact angle decreased to 41.3 and $0^{\circ}$ as the process time increased to 15 and $60 \mathrm{~s}$. However, for the $\mathrm{N}_{2}$ sample, the contact angle decreased to $61.2^{\circ}$ when the process time is $15 \mathrm{~s}$, then, there is no variation of the angle when the process time further increased. Therefore, to reduce the plasma damage of the samples coming from the long exposure of the plasma, it is reasonable to determine the plasma process time as $60 \mathrm{~s}$ for $\mathrm{O}_{2}$ and $15 \mathrm{~s}$ for $\mathrm{N}_{2}$ samples to obtain high quality Ag NWs layer. The corresponding surface energy can be calculated using the following equation [10-12],

$$
\cos \theta=-1+2 \sqrt{\frac{\gamma_{s v}}{\gamma_{l v}}} e^{-\beta\left(\gamma_{l v}-\gamma_{s v}\right)^{2}}
$$

where $\theta$ is the contact angle, $\gamma_{s v}$ is the PDMS surface tension, $\gamma_{v}$ is the DI water surface tension, and $\beta$ is the exponential exponent. The calculated values of surface energy were about $20.2,46.6$, and $72.2 \mathrm{~mJ} / \mathrm{m}^{2}$, respectively. Accordingly, as the surface angle decreased, corresponding surface energy increased when $\mathrm{O}_{2}$ and $\mathrm{N}_{2}$ plasma treatments are applied.

Figures 2(a) and (b) show the microscopy images of the spray coated Ag NWs. Depending on the surface contact angle and surface energy, the Ag NWs coated on the different plasma-treated substrate show large differences. The $\mathrm{O}_{2}$ plasma treated sample shows the best quality and good coverage with the lowest sheet resistances. Initial sheet resistances of the reference, $\mathrm{N}_{2}$, and $\mathrm{O}_{2}$ samples were about 900,660 , and $3.0 \Omega /$ square, respectively. It is noted that to obtain the best Ag NWs film quality, sufficient low contact angle and high surface energy condition should be satisfied because the sheet resistance of $\mathrm{O}_{2}$ sample was up to about 400 times smaller than those of other samples. Though the $\mathrm{N}_{2}$ plasma treated sample shows low contact angle compared to the reference sample, the uniformity and the coverage of the Ag NWs film on the $\mathrm{N}_{2}$ sample is very poor and its initial resistance (strain $=0 \%$ ) is similar with that of the reference sample. Therefore, the Ag NWs film quality is solely dependent on the contact angle and the surface energy, which means the $\mathrm{N}_{2}$ atom is inactive to 
(a)
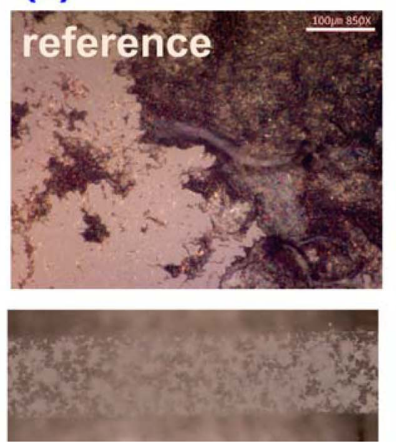

(b)
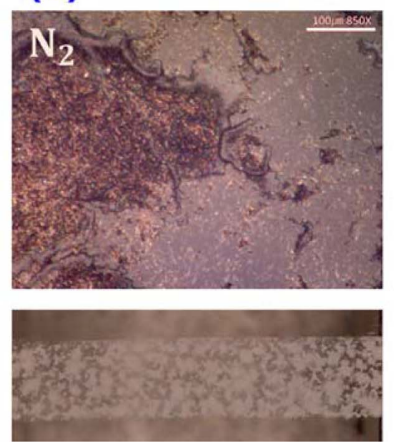

(c)
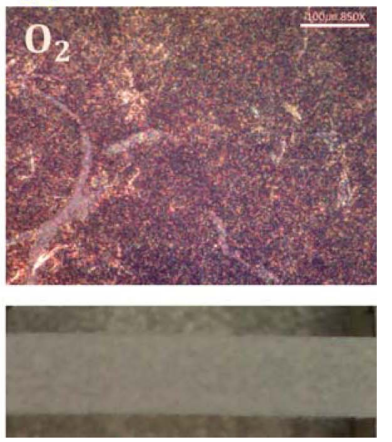

Figure 2. Microscopy images of (a) reference, (b) $\mathrm{N}_{2}$, and (c) $\mathrm{O}_{2}$ samples.

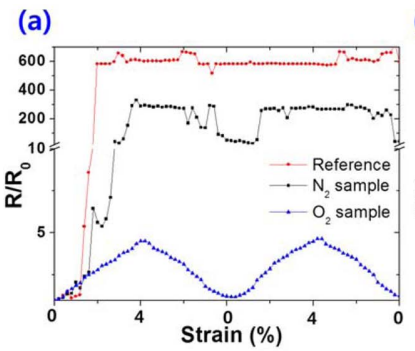

(b)

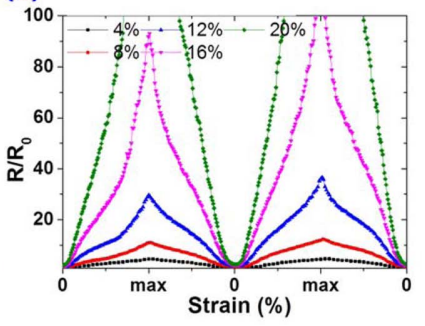

Figure 3. (a) $R / R_{0}$ vs. strain for maximum strain of $4 \%$ (all samples). (b) $R / R_{0}$ vs. strain for maximum strain of $20 \%$ for the $\mathrm{O}_{2}$ sample.
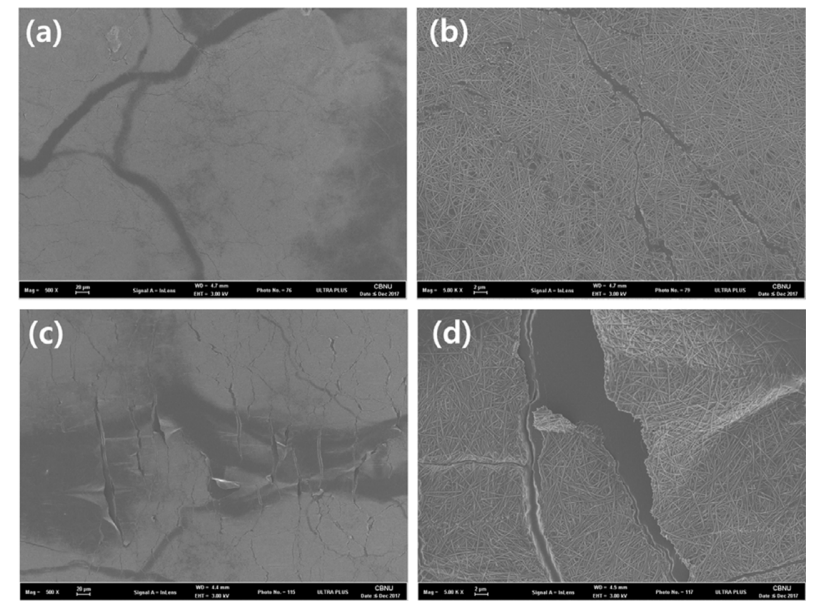

Figure 4. SEM images of Ag NWs on the PDMS substrate when (a) strain $=0 \%$ (low magnitude), (b) strain $=0 \%$ (high magnitude), (c) strain=20\% (low magnitude), and (d) strain=20\% (high magnitude).

the PDMS substrate.

Then, we performed two cycles stretching measurements with the maximum strain of $20 \%$ at stretching speed of $0.5 \mathrm{~mm} / \mathrm{s}$. Figure 3 (a) shows sheet resistance variations of the reference, $\mathrm{N}_{2}$, and $\mathrm{O}_{2}$ samples for $4 \%$ maximum strain. The sheet resistances (R) were normalized by initial resistance $\left(\mathrm{R}_{0}\right)$ when stretched. It was observed that the reference and $\mathrm{N}_{2}$ sample shows no stretching capability. The $R / R_{0}$ values steeply increased for a very small strain. However, the $\mathrm{O}_{2}$ sample shows good conductivity and the maximum $\mathrm{R} / \mathrm{R}_{0}$ value was under $5(\Omega / \Omega)$. Figure $3(\mathrm{~b})$ shows sheet resistance variations for the $\mathrm{O}_{2}$ sample as the maximum strain increased up to $20 \%$. For a large strain, the $\mathrm{O}_{2}$ sample shows further increases of $\mathrm{R} / \mathrm{R}_{0}$ values. In example, at $16 \%$ maximum strain, the $R / R_{0}$ value increased up to $90(\Omega / \Omega)$. However, the sample shows still conductivity with finite values of the sheet resistance (under $100 \Omega$ / square for $16 \%$ maximum strain). This indicates that the Ag NWs show sufficient feasibility to fabricate the stretchable electrodes.

To verify the surface morphologies when stretched, scanning electron microscopy (SEM) images were obtained for 0 and $20 \%$ strains. Figures 4 (a) to (d) show the surface SEM images for $0 \%$ strain ((a) and (b)), and $20 \%$ strain ((c) and (d)), respectively. There are many random cracks for $0 \%$ strain, which are originated from the high substrate temperature when spray coating process, as shown in Figs. 4(a) and (b). For a large strain (20\% strain), many vertical cracks are generated perpendicular to the stretching direction originated from the external strain, as shown in Figs. 4(c) and (d). It should be noted that the mechanical rupture of Ag NWs film are similar with that of rigid film and the cracks make 'open circuits' regions in the network of the Ag NWs, which cause the increase of the resistance. This is because the Ag NWs solution contains PVP stabilizer. However, the film still shows reasonable conductivity under $100 \Omega$ /square up to $16 \%$ strain indicating that it can be used as the stretchable electrodes applications.

\section{Summary}

In this paper, the stretching capabilities of Ag NWs film depending on the plasma treatments $\left(\mathrm{N}_{2}\right.$ and $\left.\mathrm{O}_{2}\right)$ conditions were analyzed. The $\mathrm{O}_{2}$ sample shows the best performance with the lowest sheet resistance of $3.0 \Omega /$ square due to low surface energy after the treatments. The stretching capability measurements indicate that the $\mathrm{O}_{2}$ sample can be used as stretchable electrodes because it shows finite resistance under $100 \Omega /$ square for $16 \%$ strain conditions. It is clearly shown that low resistance and reasonable stretching capability can be achieved using the Ag NWs electrodes. 


\section{Acknowledgements}

This research was supported by a National Research Foundation of Korea (NRF) grant funded by the Korean government (No. NRF-2017R1D1A1B03035271). This research was also supported by the Ministry of Science and ICT (MSIT), Korea, under the Information Technology Research Center (ITRC) support program (IITP-20172015-0-00448) supervised by the Institute for Information \& Communications Technology Promotion (IITP).

\section{References}

[1] J. Jeong and J. Jeong, Jpn. J. Appl. Phys. 56, 115801 (2017)

[2] E. P. Chan and A. J. Crosby, Soft Mater. 2, 324 (2006).
[3] S. Lee and J. Jeong, Current Applied Physics 17, 1727 (2017).

[4] S. Chung, J. Lee, H. Song, S. Kim, J. Jeong, and Y. Honga, Appl. Phys. Lett. 98, 153110 (2011).

[5] X. Nie, H. Wang, and J. Zou, Appl. Surf. Sci. 261, 554 (2012).

[6] D. Y. Choi, H. W. Kang, H. J. Sung, and S. S. Kim, Nanoscale 5, 977 (2013).

[7] N. Munkhbaatar, I. Ryu, D. Park and S. Yim, Appl. Sci. Converg. Technol. 24, 219 (2015).

[8] J. S. Oh and G. Y. Yeom, Appl. Sci. Converg. Technol. 26, 149 (2017).

[9] J. Park, T. Kim, D.Stryakhilev, J. Lee, S. An, Y. Pyo, D. Lee, Y. G. Mo, D. Jin, and H. K. Chung, Appl. Phys. Lett. 95, 013503 (2009).

[10] H. Tavana and A. W. Neumann, Adv. Colloid Interface Sci. 132, 1 (2007).

[11] B. Mosadegh, H. Tavana, S. C. Lesher-Perez, and S. Takayama, Lab Chip 11, 738 (2011).

[12] T. Yamada, Y. Hayamizu, Y. Yamamoto, Y. Yomogida, A. I. Najafabadi, D. N. Futaba, and K. Hata, Nat. Nanotechnol. 6, 296 (2011). 